\title{
Research Article \\ Permanence for a Delayed Nonautonomous SIR Epidemic Model with Density-Dependent Birth Rate
}

\author{
Li Yingke, ${ }^{1}$ Chen Liang, ${ }^{2}$ and Wang $\mathrm{Kai}^{3}$ \\ ${ }^{1}$ College of Mathematics and Physics, Xinjiang Agricultural University, Urumqi 830052, China \\ ${ }^{2}$ Department of Mathematics, Changji College, Changji 831100, China \\ ${ }^{3}$ Department of Medical Engineering and Technology, Xinjiang Medical University, Urumqi 830054, China
}

Correspondence should be addressed to Li Yingke, lykhefeng@yahoo.com.cn

Received 21 February 2011; Accepted 12 May 2011

Academic Editor: Ibrahim Yalcinkaya

Copyright (C) 2011 Li Yingke et al. This is an open access article distributed under the Creative Commons Attribution License, which permits unrestricted use, distribution, and reproduction in any medium, provided the original work is properly cited.

Based on some well-known SIR models, a revised nonautonomous SIR epidemic model with distributed delay and density-dependent birth rate was considered. Applying some classical analysis techniques for ordinary differential equations and the method proposed by Wang (2002), the threshold value for the permanence and extinction of the model was obtained.

\section{Introduction}

For understanding the spread of infectious diseases in population, mathematical models that use the theories of ordinary differential equations in epidemiology have been developed rapidly. Epidemic models with delay, including either the autonomous continuous systems or the discrete ones, were discussed by many authors [1-13]. The important subjects for this models are looking for the threshold value that determines whether the infectious disease will be permanent or extinct. Hence, permanence of disease plays an important role in epidemiology. Furthermore, it is well known that models with distributed delay are more appropriate than the discrete ones because it is considered more realistic to assume the infectivity to be a function of the duration since infection and up to some maximum duration.

Recently, Song and Ma in [4] and Song et al. in [5] discussed the permanence of disease in a generalized autonomous SIR epidemic model with density dependent birth rate. On the other hand, for the nonautonomous systems [1] (and the reference therein), the literature is still very inadequate. Motivated by the works in $[1,2,4,5,9,10]$, in this paper, we will 
consider the following nonautonomous delayed systems with density-dependent birth rate:

$$
\begin{aligned}
& \dot{S}(t)=-\beta(t) S(t) \int_{0}^{h} I(t-s) d \eta(s)-\mu_{1}(t) S(t)+b(t)\left[1-\beta_{1}(t) \frac{N(t)}{1+N(t)}\right], \\
& \dot{I}(t)=\beta(t) S(t) \int_{0}^{h} I(t-s) d \eta(s)-\mu_{2}(t) I(t)-\lambda(t) I(t), \\
& \dot{R}(t)=\lambda(t) I(t)-\mu_{3}(t) R(t),
\end{aligned}
$$

where $N(t)=S(t)+I(t)+R(t)$ denote the total number of a population at time $t, S$ is the susceptible population, $I$ is the infective population and $R$ is the removed population. It is assumed that all newborns are susceptible. Functions $\mu_{1}(t), \mu_{2}(t)$ and $\mu_{3}(t)$ are instantaneous per capita mortality rates of susceptible, infective, and recovered population, respectively; functions $b(t)$ and $\lambda(t)$ represent the birth rate of the population and the recovery rate of infectives, respectively; function $\beta_{1}(t)\left(0 \leq \beta_{1}(t)<1\right)$ reflects the relation between the birth rate and the density of population. The nonnegative constant $h$ is the time delay. The function $\eta(s):[0, h] \rightarrow[0,+\infty)$ is nondecreasing and has hounded variation such that

$$
\int_{0}^{h} d \eta(s)=\eta(h)-\eta(0)=1
$$

\section{Preliminaries}

Firstly, we give some notations for convenience: if $f(t)$ is a continuous bounded function defined on $[0,+\infty)$, then we set

$$
f^{L}=\inf _{t \geq 0} f(t), \quad f^{M}=\sup _{t \geq 0} f(t)
$$

Secondly, for system (1.1), we introduce the following assumption: functions $b(t), \beta(t)$, $\beta_{1}(t), \lambda(t), \mu_{1}(t), \mu_{2}(t), \mu_{3}(t)$ are nonnegative continuous bounded functions and have positive lower bounds. It is biologically natural to assume that $\mu_{2}(t), \mu_{3}(t) \geq \mu_{1}(t)$ (i.e., epidemics will increase the death rates of the infective and removed).

The initial condition of (1.1) is given as

$$
S(\theta)=\varphi_{1}(\theta), \quad I(\theta)=\varphi_{2}(\theta), \quad R(\theta)=\varphi_{3}(\theta), \quad-\infty<\theta \leq 0,
$$

where $\phi=\left(\varphi_{1}, \varphi_{2}, \varphi_{3}\right)^{T} \in C$ such that $\varphi_{i}(\theta) \geq 0(i=1,2,3)$ for all $-\infty<\theta \leq 0$, and $C$ denotes the Banach space $C((-\infty, 0], \mathbb{R})$ of bounded continuous functions mapping the interval $(-\infty, 0]$ into $\mathbb{R}$ with the toplogy of uniform convergence, that is, for $\varphi \in C$, we designate the norm of an element $\phi \in C$ by $\|\phi\|=\sup _{-\infty<\theta \leq 0}\left\{\left|\varphi_{1}(\theta)\right|,\left|\varphi_{2}(\theta)\right|,\left|\varphi_{3}(\theta)\right|\right\}$. By a biological meaning, we further assume that $\varphi_{i}(\theta)>0$ for $i=1,2,3$.

Now, a very impotent lemma for investigating the dynamics of the above system will be given. 
Lemma 2.1. Any solution $(S(t), I(t), R(t))$ of system (1.1) with (2.2) is positive for all $t \geq 0$.

Proof. Because the right-hand side of system (1.1) is completely continuous and locally Lipschitzian on $C$, the solution $(S(t), I(t), R(t))$ of system (1.1) with initial condition (2.2) exists and is unique on $[0, \alpha)$, where $0<\alpha \leq+\infty$. Firstly, we show that $S(t)>0$ for all $t \in[0, \alpha)$. Otherwise, there is a $t_{1} \in(0, \alpha)$ such that $S\left(t_{1}\right)=0, \dot{S}\left(t_{1}\right) \leq 0$, and $S(t)>0$ for all $t \in\left[0, t_{1}\right)$. Hence, we have $I(t)>0$ for all $t \in\left[0, t_{1}\right)$. If the statement is not true, then there is a $t_{2} \in\left(0, t_{1}\right)$ such that $I\left(t_{2}\right)=0, \dot{I}\left(t_{2}\right) \leq 0$, and $I(t)>0$ on $t \in\left[0, t_{2}\right)$. Integrating the second equation of (1.1) from 0 to $t_{2}$, we get

$$
I\left(t_{2}\right)=I(0) e^{-\int_{0}^{t_{2}}\left[\mu_{2}(s)+\gamma(s)\right] d s}+\int_{0}^{t_{2}} \int_{0}^{h} \beta(u) S(u) I(u-\rho) e^{\int_{t_{2}}^{u}\left[\mu_{2}(s)+\gamma(s)\right] d s} d \rho d u>0,
$$

which is a contradiction. This shows that $I(t)>0$ for all $t \in\left[0, t_{1}\right)$. From the third equation of (1.1), we also get that

$$
R(t) \geq R(0) \exp \left\{-\int_{0}^{t_{2}}\left[\mu_{3}(s)\right] d s\right\}>0
$$

on $\left[0, t_{1}\right)$. Therefore, from the first equation of system (1.1)

$$
\dot{S}\left(t_{1}\right)=b\left(t_{1}\right)\left[1-\beta_{1}\left(t_{1}\right)\right]+\frac{b\left(t_{1}\right) \beta_{1}\left(t_{1}\right)}{1+I\left(t_{1}\right)+R\left(t_{1}\right)}>0,
$$

which also is a contradiction. This shows that $S(t)>0$ on $[0, \alpha)$. By the above calculation, it is obvious that $I(t)>0, R(t)>0$ for all $t \in[0, \alpha)$.

\section{Permanence and Extinction}

In this section, we investigate the permanence of system (1.1) and demonstrate how the disease will be extinct under some conditions.

Theorem 3.1. System (1.1) is permanent if

$$
S^{0}=\frac{\left[b^{M}\left(1-\beta_{1}\right)^{M}-\mu_{1}^{L}\right]+\sqrt{\left[b^{M}\left(1-\beta_{1}\right)^{M}-\mu_{1}^{L}\right]^{2}+4 \mu_{1}^{L} b^{M}}}{2 \mu_{1}^{L}}>\left(\frac{\mu_{2}+\lambda}{\beta}\right)^{M} .
$$

Proof. We will give the following several propositions to complete the proof of this theorem.

Proposition 3.2. Assume that (3.1) holds. Then

$$
\limsup _{t \rightarrow+\infty} N(t) \leq S^{0}
$$


Proof. From system (1.1), we have that

$$
\begin{aligned}
\dot{N}(t) & =-\mu_{1}(t) S(t)-\mu_{2}(t) I(t)-\mu_{3}(t) R(t)+b(t)\left[1-\beta_{1}(t)\right]+\frac{b(t) \beta_{1}(t)}{1+N(t)} \\
& \leq \frac{1}{1+N(t)}\left\{-\mu_{1}(t) N(t)[1+N(t)]+b(t)\left[1-\beta_{1}(t)\right][1+N(t)]+b(t) \beta_{1}(t)\right\} \\
& \leq \frac{1}{1+N(t)}\left\{-\mu_{1}^{L} N^{2}(t)-\mu_{1}^{L} N(t)+b^{M}\left[1-\beta_{1}\right]^{M} N(t)+b^{M}\right\} \\
& =-\frac{1}{1+N(t)}\left[N(t)-S^{0}\right]\left[N(t)-S_{*}^{0}\right],
\end{aligned}
$$

where

$$
S_{*}^{0}=\frac{\left[b^{M}\left(1-\beta_{1}\right)^{M}-\mu_{1}^{L}\right]-\sqrt{\left[b^{M}\left(1-\beta_{1}\right)^{M}-\mu_{1}^{L}\right]^{2}+4 \mu_{1}^{L} b^{M}}}{2 \mu_{1}^{L}}<0 .
$$

Now, we consider only the following three cases.

(i) $N(t) \leq S^{0}$ for all sufficiently large $t$. Obviously, for the case (3.2) is true.

(ii) $N(t) \geq S^{0}$ for all sufficiently large $t$. In this case, $\dot{N}(t) \leq 0$ for all sufficiently large $t$ from (3.2). Hence, there is a constant $\widetilde{N}$ such that

$$
\lim _{t \rightarrow+\infty} N(t)=\widetilde{N} \geq S^{0}
$$

In fact, we can obtain that $\widetilde{N}=S^{0}$. If $\widetilde{N}>S^{0}$, for all sufficiently small $\varepsilon: 0<\varepsilon<\widetilde{N}-S^{0}$, there is a sufficiently large $t^{*}$ such that for any $t>t^{*}$,

$$
0<\widetilde{N}-\varepsilon \leq N(t) \leq \widetilde{N}+\varepsilon
$$

From (3.3), we have that for all $t \geq t^{*}$,

$$
\dot{N}(t) \leq-\frac{1}{1+\widetilde{N}+\varepsilon}\left(\widetilde{N}-S^{0}-\varepsilon\right)\left(\widetilde{N}-S_{*}^{0}-\varepsilon\right)<0,
$$

which implies that $\lim _{t \rightarrow+\infty} N(t)=-\infty$. This is a contradiction to $\lim _{t \rightarrow+\infty} N(t)=\widetilde{N}$. Hence, $\widetilde{N}=S^{0}$.

(iii) There is a time sequence $\left\{t_{n}\right\}: t_{n} \rightarrow+\infty$ as $n \rightarrow+\infty$ such that $N\left(t_{n}\right)>S^{0}$ and $\dot{N}\left(t_{n}\right)=0$. Then, for $t=t_{n}$

$$
\dot{N}(t)=-\frac{1}{1+N(t)}\left[N(t)-S^{0}\right]\left[N(t)-S_{*}^{0}\right]<0,
$$

which is a contradiction to $\dot{N}\left(t_{n}\right)=0$. This completes the proof. 
Proposition 3.3. Assume that (3.1) holds. Then any positive solution $(S(t), I(t), R(t))$ of system (1.1) with (2.2) satisfies

$$
\liminf _{t \rightarrow+\infty} S(t) \geq\left(\frac{b\left(1-\beta_{1}\right)}{\beta S^{0}+\mu_{1}}\right)^{L} \equiv v_{1}
$$

Proof. For any sufficiently small $\varepsilon>0$, from Proposition 3.2, there is a large $t_{1}>0$ such that for all $t>t_{1}>0, I(t) \leq S^{0}+\varepsilon$. Hence, for $t>t_{1}+h$,

$$
\begin{aligned}
\dot{S}(t) & \geq-\beta(t) S(t) \int_{0}^{h} I(t-s) d \eta(s)-\mu_{1}(t) S(t)+b(t)\left[1-\beta_{1}(t)\right] \\
& \geq b(t)\left[1-\beta_{1}(t)\right]-\left[\mu_{1}(t)+\beta(t)\left(S^{0}+\varepsilon\right)\right] S(t),
\end{aligned}
$$

which clearly implies that

$$
\liminf _{t \rightarrow+\infty} S(t) \geq\left(\frac{b\left(1-\beta_{1}\right)}{\beta\left(S^{0}+\varepsilon\right)+\mu_{1}}\right)^{L} \equiv v_{1} .
$$

Noting that $\varepsilon$ can be arbitrarily small, the conclusion is valid. This completes the proof of Proposition 3.3.

Proposition 3.4. Assume that (3.1) holds. Then for any positive solution $(S(t), I(t), R(t))$ of system (1.1) with (2.2), one has

$$
\liminf _{t \rightarrow+\infty} I(t) \geq \rho e^{-\left(\mu_{1}+\lambda\right)^{M} d} \equiv v_{2}
$$

where $\rho>0, \varepsilon_{0}>0$ and $d>0$ satisfy

$$
\begin{aligned}
p & =\frac{1}{\left[\mu_{1}+\rho \beta^{M}\right]^{M}}\left\{b\left[1-\beta_{1}\right]+\frac{b \beta_{1}}{1+S^{0}+\varepsilon_{0}}\right\}^{L}>\left(\frac{\mu_{2}+\lambda}{\beta}\right)^{M}, \\
S^{\Delta} & =p\left\{1-\exp \left[-\left(\mu_{1}^{L}+\rho \beta^{M}\right) d\right]\right\}>\left(\frac{\mu_{2}+\lambda}{\beta}\right)^{M} .
\end{aligned}
$$

Proof. By (3.3) and (3.1) it is obvious that

$$
S^{0}=\frac{1}{\mu_{1}^{L}}\left\{\left[b\left(1-\beta_{1}\right)\right]^{M}+\frac{\left(b \beta_{1}\right)^{M}}{1+S^{0}}\right\}>\left(\frac{\mu_{2}+\lambda}{\beta}\right)^{M} .
$$

Then there exist two positive constants $\rho>0, \varepsilon_{0}>0$ and $d>0$ such that $p>\left(\left(\mu_{2}+\lambda\right) / \beta\right)^{M}$ and $S^{\Delta}>\left(\left(\mu_{2}+\lambda\right) / \beta\right)^{M}$. 
Let us consider the following differential function $V(t)$ :

$$
V(t)=I(t)+\int_{0}^{h} \int_{t-s}^{t} \beta(u+s) S(u+s) I(u) d u d \eta(s) .
$$

Then, the derivative of $V(t)$ along with the solution of (1.1) with (2.2) is

$$
\begin{aligned}
\dot{V}(t) & =\dot{I}(t)+\int_{0}^{h} \beta(t+s) S(t+s) I(t) d \eta(s)-\int_{0}^{h} \beta(t) S(t) I(t-s) d \eta(s) \\
& =\left\{\int_{0}^{h} \beta(t+s) S(t+s) d \eta(s)-\left[\mu_{2}(t)+\lambda(t)\right]\right\} I(t) \\
& \geq\left\{\beta^{L} \int_{0}^{h} S(t+s) d \eta(s)-\left(\mu_{2}+\lambda\right)^{M}\right\} I(t) .
\end{aligned}
$$

We claim that it is impossible that $I(t) \leq \rho$ for all $t \geq t_{1}$ ( $t_{1}$ is any nonnegative constant). In fact, supposing the contrary, it follows from the first equation of (1.1) and (3.1) that for any $t \geq t_{1}+h$,

$$
\begin{aligned}
\dot{S}(t) & =-\beta(t) S(t) \int_{0}^{h} I(t-s) d \eta(s)-\mu_{1}(t) S(t)+b(t)\left[1-\beta_{1}(t)\right]+\frac{b(t) \beta_{1}(t)}{1+N(t)} \\
& \geq b_{1}(t)\left[1-\beta_{1}(t)\right]+\frac{b(t) \beta_{1}(t)}{1+S^{0}+\varepsilon_{0}}-\left[\mu_{1}(t)+\rho \beta^{M}\right] S(t) .
\end{aligned}
$$

Integrating the above inequality from $t_{1}+h$ to $t$, we obtain

$$
\begin{aligned}
S(t) \geq & S\left(t_{1}+h\right) \exp \left[\int_{t}^{t_{1}+h}\left(\mu_{1}(s)+Q \beta^{M}\right) d s\right] \\
& +\int_{t_{1}+h}^{t}\left\{b(t)\left[1-\beta_{1}(t)\right]+\frac{b(t) \beta_{1}(t)}{1+S^{0}+\varepsilon_{0}}\right\} \exp \left\{\int_{t}^{s}\left[\mu_{1}(\tau)+Q \beta^{M}\right] d \tau\right\} d s \\
\geq & \frac{\int_{t_{1}+h}^{t}\left\{b(t)\left[1-\beta_{1}(t)\right]+b(t) \beta_{1}(t) /\left(1+S^{0}+\varepsilon_{0}\right)\right\}\left[\mu_{1}(\tau)+Q \beta^{M}\right] \exp \left\{\int_{0}^{s}\left[\mu_{1}(\tau)+Q \beta^{M}\right] d \tau\right\} d s}{\left[\mu_{1}(\tau)+Q \beta^{M}\right] \exp \left\{\int_{0}^{t}\left[\mu_{1}(\tau)+Q \beta^{M}\right] d \tau\right\}} \\
\geq & \frac{1}{\left(\mu_{1}+Q \beta^{M}\right)^{M}}\left\{b\left[1-\beta_{1}\right]+\frac{b \beta_{1}}{1+S^{0}+\varepsilon_{0}}\right\}^{L}\left\{1-\exp \left[-\left(\mu_{1}^{L}+\rho \beta^{M}\right)\left(t-t_{1}-h\right)\right]\right\} .
\end{aligned}
$$


Thus, for any $t \geq t_{1}+h+d$,

$$
S(t) \geq \frac{1}{\left[\mu_{1}+Q \beta^{M}\right]^{M}}\left\{b\left[1-\beta_{1}\right]+\frac{b \beta_{1}}{1+S^{0}+\varepsilon_{0}}\right\}^{L}\left\{1-\exp \left[-\left(\mu_{1}^{L}+\rho \beta^{M}\right) d\right]\right\}=S^{\Delta} .
$$

Then, by the above inequality, from (3.16) and (3.19), we get, for any $t \geq t_{1}+h+d$,

$$
\dot{V}(t) \geq\left[\beta^{L} S^{\Delta}-\left(\mu_{2}+\lambda\right)^{M}\right] I(t)
$$

Set

$$
\underline{i}=\min _{\theta \in[-h, 0]} I\left(t_{1}+d+2 h+\theta\right)
$$

Next, we will prove that $I(t) \geq \underline{i}$ for all $t \geq t_{1}+d+h$. Suppose that this is not true. Then there is a $T \geq 0$ such that $I(t) \geq \underline{i}$ for all $t \in\left[t_{1}+d+h, t_{1}+d+2 h+T\right], I\left(t_{1}+d+2 h+T\right)=\underline{i}$ and $\dot{I}\left(t_{1}+d+2 h+T\right) \leq 0$. On the other hand, by the second equation of (1.1) and (3.16), for $t=t_{1}+d+2 h+T$,

$$
\begin{aligned}
\dot{I}(t) & =\beta(t) S(t) \int_{0}^{h} I(t-s) d \eta(s)-\mu_{2}(t) I(t)-\lambda(t) I(t) \\
& \geq\left[\beta^{L} S^{\Delta}-\left(\mu_{2}+\lambda\right)^{M}\right] \underline{i}>0 .
\end{aligned}
$$

This is a contradiction to $\dot{I}\left(t_{1}+d+2 h+T\right) \leq 0$. Hence, $I(t) \geq \underline{i}$ for all $t \geq t_{1}+d+h$.

Consequently, for all $t \geq t_{1}+d+h$, we obtain that

$$
\dot{V}(t) \geq\left[\beta^{L} S^{\Delta}-\left(\mu_{2}+\lambda\right)^{M}\right] \underline{i}>0,
$$

which implies that $V(t) \rightarrow+\infty$ as $t \rightarrow+\infty$. In fact, from Proposition 3.2, V(t) is bounded, which is a contradiction. Consequently, the claim is proved. From the claim, we will discuss the following possible cases:

(i) $I(t) \geq \rho$ for all large $t$;

(ii) $I(t)$ oscillates about $\rho$ for all large $t$.

We show that $I(t) \geq \rho \mathrm{e}^{-\left(\mu_{2}+\lambda\right)^{M}(d+h)}$ for all large $t$. Evidently, we only need to consider case (ii). Let $t_{1}$ and $t_{2}$ be sufficiently large such that

$$
\begin{gathered}
I\left(t_{1}\right)=I\left(t_{2}\right)=\rho, \\
I(t)<\rho \quad \text { as } t \in\left(t_{1}, t_{2}\right) .
\end{gathered}
$$


If $t_{2}-t_{1} \leq d+h$ and from the second equation of (1.1) we have that $\dot{I}(t) \geq-\left(\mu_{2}+\lambda\right)^{M} I(t)$, it is obvious that $I(t) \geq \rho e^{-\left(\mu_{2}+\lambda\right)^{M}(d+h)}$ for $t \in\left(t_{1}, t_{2}\right)$; if $t_{2}-t_{1}>d+h$, and then it is clear that $I(t) \geq \rho e^{-\left(\mu_{2}+\lambda\right)^{M}(d+h)}$ for $t \in\left[t_{1}+d+h, t_{2}\right]$; in fact, if not, there exists a $T^{*} \geq 0$, such that $I(t) \geq \rho e^{-\left(\mu_{2}+\lambda\right)^{M}(d+h)}$ for $t \in\left[t_{1}, t_{1}+d+h+T^{*}\right] \subseteq\left[t_{1}, t_{2}\right], I\left(t_{1}+d+h+T^{*}\right)=\rho e^{-\left(\mu_{2}+\lambda\right)^{M}(d+h)}$ and $\dot{I}\left(t_{1}+d+h+T^{*}\right) \leq 0$. Using the second equation of system (1.1), as $t=t_{1}+d+h+T^{*}$,

$$
\begin{aligned}
\dot{I}(t) & =\beta(t) S(t) \int_{0}^{h} I(t-s) d \eta(s)-\mu_{2}(t) I(t)-\lambda(t) I(t) \\
& \geq\left[\beta^{L} S^{\Delta}-\left(\mu_{2}+\lambda\right)^{M}\right] \rho e^{-\left(\mu_{2}+\lambda\right)^{M}(d+h)}>0 .
\end{aligned}
$$

This is a contradiction to $\dot{I}\left(t_{1}+d+h+T^{*}\right) \leq 0$. So, $I(t) \geq \rho e^{-\left(\mu_{2}+\lambda\right)^{M}(d+h)}$ is valid for all $t \in\left[t_{1}, t_{2}\right]$. Since this kind of interval $\left[t_{1}, t_{2}\right]$ is chosen as an arbitrary way,

$$
\liminf _{t \rightarrow+\infty} I(t) \geq \rho e^{-\left(\mu_{2}+\lambda\right)^{M}(d+h)}=v_{2}>0
$$

The proof of Proposition 3.4 is completed.

Form the third equation of system (1.1) and Proposition 3.4, we easily obtain

$$
\liminf _{t \rightarrow+\infty} R(t) \geq v_{2}\left(\frac{\lambda}{\mu_{2}}\right)^{L}=v_{3}>0
$$

Thus, the system (1.1) is permanent by Propositions 3.2-3.4. The proof of Theorem 3.1 is completed.

Next, we give a useful lemma discussing the extinction of the disease.

Lemma 3.5 (see [12]). Consider an autonomous system of delayed differential equation

$$
\dot{x}(t)=a_{1} \int_{0}^{h} x(t-h) d \eta(s)-a_{2} x(t),
$$

where $a_{1}, a_{2}$ are two constants. If $0 \leq a_{1}<a_{2}$, then for any solutions $x(t)$ with initial condition $\phi(\theta) \geq 0, \theta \in[-h, 0]$, one has

$$
\lim _{t \rightarrow+\infty} x(t)=0 .
$$


Theorem 3.6. If

$$
S^{0}<\left(\frac{\mu_{2}+\lambda}{\beta}\right)^{L}
$$

then $\lim _{t \rightarrow+\infty} x(t)=0$; that is, the disease in system (1.1) will be extinct.

Proof. From Lemma 3.5, for any sufficiently small $\varepsilon>0$, there exists a $T>0$, for $t>T$, such that

$$
S(t)<S^{0}+\varepsilon
$$

Therefore, from the second equation of (1.1), we have

$$
\begin{aligned}
\dot{I}(t) & =\beta(t) S(t) \int_{0}^{h} I(t-s) d \eta(s)-\left[\mu_{2}(t)+\lambda(t)\right] I(t) \\
& \leq \beta^{M}\left(S^{0}+\varepsilon\right) \int_{0}^{h} I(t-s) d \eta(s)-\left[\mu_{2}+\lambda\right]^{L} I(t) .
\end{aligned}
$$

Using the comparison theorem of functional differential equations and Lemma 3.5, we can easily get $\lim _{t \rightarrow+\infty} I(t)=0$. The proof is completed.

\section{Discussion}

In this paper, we discussed a revised nonautonomous SIR epidemic model with distributed time delay and density-dependent birth rate. Theorems 3.1 and 3.6 show that the disease will be permanent when $S^{0}>\left(\left(\mu_{2}+\lambda\right) / \beta\right)^{M}$, and the disease will be extinct when $S^{0}<$ $\left(\left(\mu_{2}+\lambda\right) / \beta\right)^{L}$. From the point of view of biology, Theorems 3.1 and 3.6 suggest that the inequality $S^{0}>\left(\left(\mu_{2}+\lambda\right) / \beta\right)^{M}$ may be the threshold value for an epidemic disease to occur. The technique for obtaining the sufficient condition on the permanence of the disease in this paper is the same as that in $[4,5]$ if all coefficients in system (1.1) are constants and the delay is discrete.

\section{Acknowledgment}

The authors are grateful to the anonymous referee for his/her many valuable comments and suggestions.

\section{References}

[1] T. Zhang and Z. Teng, "Global behavior and permanence of SIRS epidemic model with time delay," Nonlinear Analysis, vol. 9, no. 4, pp. 1409-1424, 2008.

[2] W. Wang, "Global behavior of an SEIRS epidemic model with time delays," Applied Mathematics Letters, vol. 15, no. 4, pp. 423-428, 2002.

[3] M. Sekiguchi and E. Ishiwata, "Global dynamics of a discretized SIRS epidemic model with time delay," Journal of Mathematical Analysis and Applications, vol. 371, no. 1, pp. 195-202, 2010. 
[4] M. Song and W. Ma, "Asymptotic properties of a revised SIR epidemic model with density dependent birth rate and time delay," Dynamics of Continuous, Discrete and Impulsive Systems. Series A: Mathmatical Analysis, vol. 13, no. 2, pp. 199-208, 2006.

[5] M. Song, W. Ma, and Y. Takeuchi, "Permanence of a delayed SIR epidemic model with density dependent birth rate," Journal of Computational and Applied Mathematics, vol. 201, no. 2, pp. 389-394, 2007.

[6] Y. Enatsu and Y. Nakata, "Global stability for a class of discrete SIR epidemic models," Mathematical Biosciences and Engineering, vol. 7, no. 2, pp. 347-361, 2010.

[7] C. C. McCluskey, "Complete global stability for an SIR epidemic model with delay-distributed or discrete," Nonlinear Analysis, vol. 11, no. 1, pp. 55-59, 2010

[8] M. Sekiguchi, "Permanence of a discrete SIRS epidemic model with time delays," Applied Mathematics Letters, vol. 23, no. 10, pp. 1280-1285, 2010.

[9] E. Beretta, T. Hara, W. Ma, and Y. Takeuchi, "Global asymptotic stability of an SIR epidemic model with distributed time delay," Nonlinear Analysis, vol. 47, no. 6, pp. 4107-4115, 2001.

[10] W. Ma and M. Song, "Global stability of an SIR epidemic model with time delay," Applied Mathematics Letters, vol. 17, no. 10, pp. 1141-1145, 2004.

[11] G. P. Samanta, "Permanence and extinction for a nonautonomous avian-human influenza epidemic model with distributed time delay," Mathematical and Computer Modelling, vol. 52, no. 9-10, pp. 17941811, 2010.

[12] T. Zhang, J. Liu, and Z. Teng, "Dynamic behavior for a nonautonomous SIRS epidemic model with distributed delays," Applied Mathematics and Computation, vol. 214, no. 2, pp. 624-631, 2009.

[13] J. Wang, J. Zhang, and Z. Jin, "Analysis of an SIR model with bilinear incidence rate," Nonlinear Analysis, vol. 11, no. 4, pp. 2390-2402, 2010. 


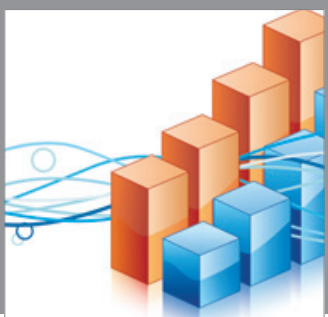

Advances in

Operations Research

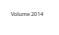

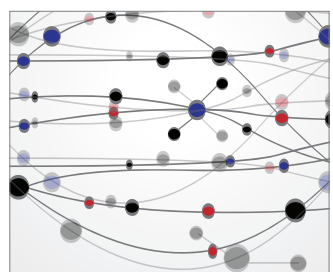

\section{The Scientific} World Journal
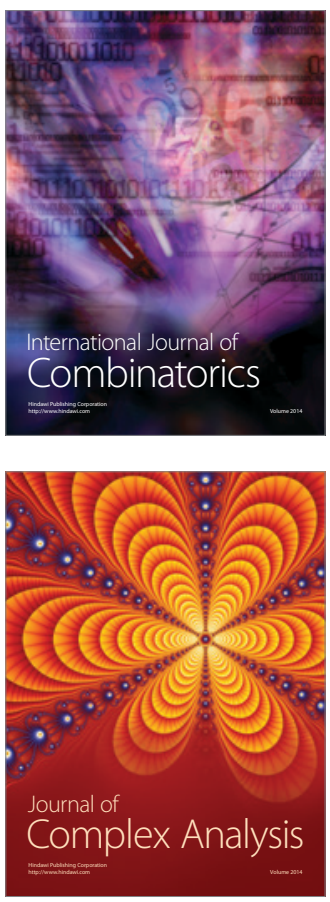

International Journal of

Mathematics and

Mathematical

Sciences
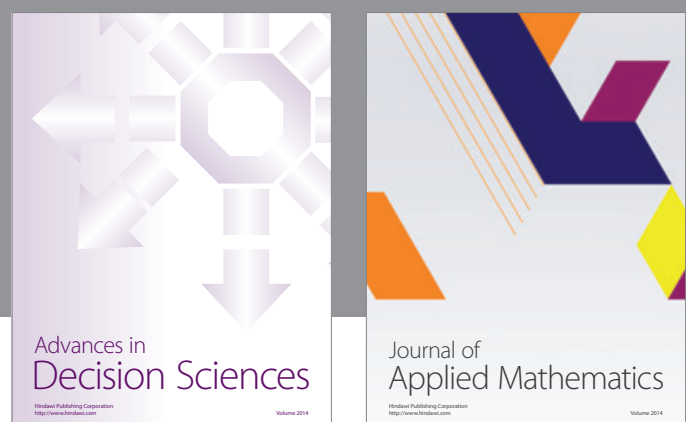

Journal of

Applied Mathematics
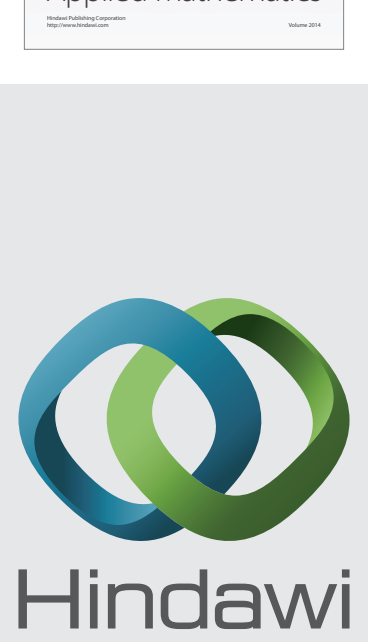

Submit your manuscripts at http://www.hindawi.com
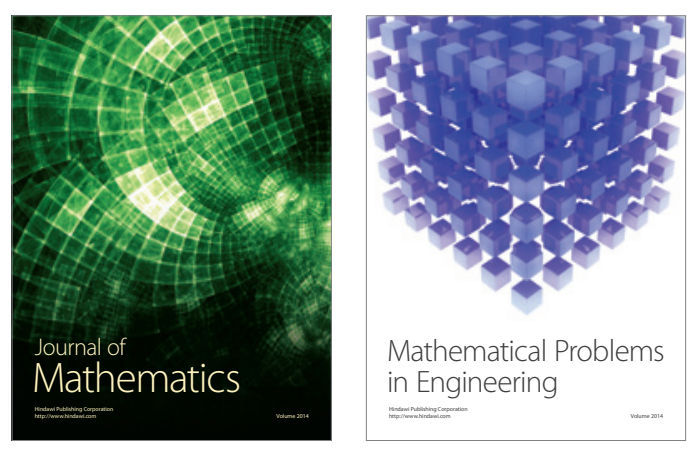

Mathematical Problems in Engineering
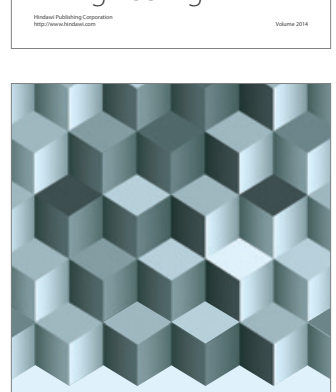

Journal of

Function Spaces
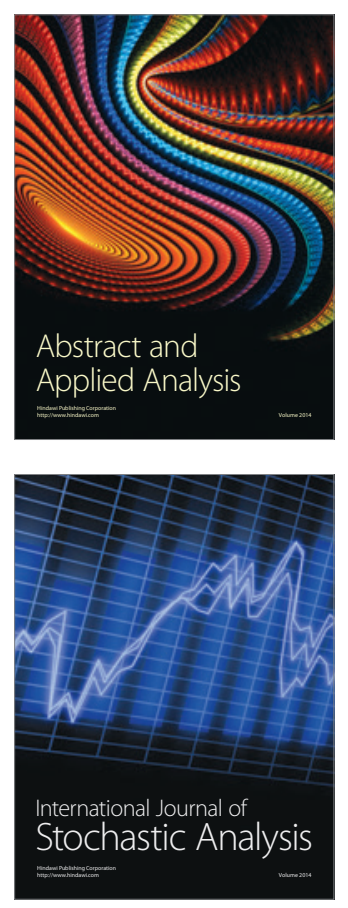

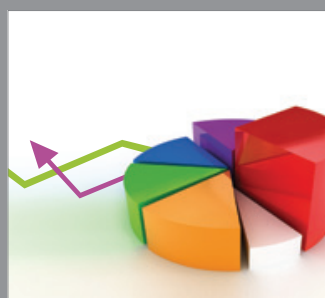

ournal of

Probability and Statistics

Promensencen
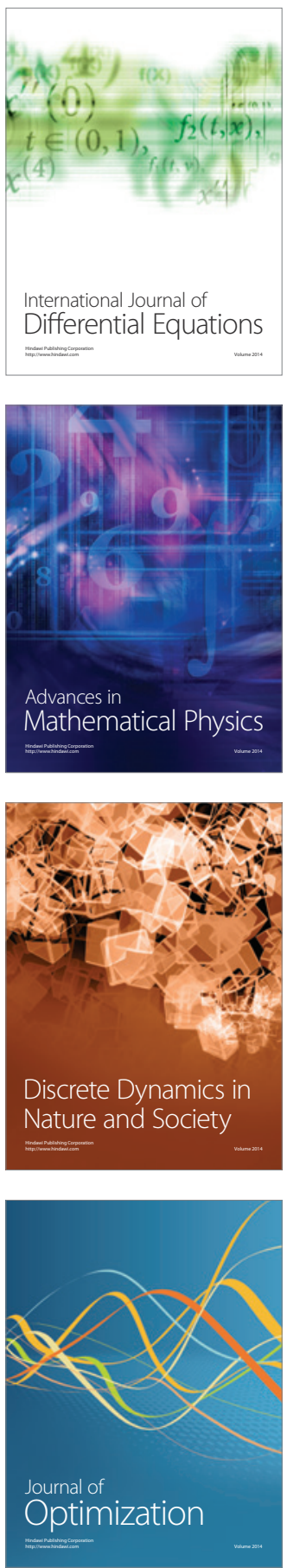